\title{
El Software Libre y las Revistas Científicas
}

\section{Open Source and Scientific Journals}

DOI: $10.46932 / \mathrm{sfjdv} 2 \mathrm{n} 2-145$

Received in: March 1st, 2021

Accepted in: May 30th, 2021

\section{Carlos Rios-Campos}

Docente de la Universidad Nacional Toribio Rodríguez de Mendoza de Amazonas. Doctor en Gestión Universitaria. Maestro en Administración. Investigador RENACYT. Bagua, Perú.

E-mail: carlos.rios@untrm.edu.pe

\section{Antero Alexander Cabrera Torres}

Docente asociado. Licenciado en Estadística, Maestría en Educación y Doctorado en Administración de la Educación. Universidad Nacional de Frontera. Sullana, Perú.

E-mail: acabrera@unf.edu.pe

\section{Freddy Manuel Camacho Delgado}

Docente Principal. Doctor en Economía. Universidad Nacional Intercultural Fabiola Salazar Leguía de Bagua. Bagua Grande, Perú.

E-mail: fcamacho@unibagua.edu.pe

\section{Jury Yesenia Aquino Trujillo}

Docente universitaria. Maestría en Ingeniería de Sistemas. Universidad Católica Santo Toribio de Mogrovejo. Chiclayo, Perú.

E-mail: jaquino@usat.edu.pe

\section{Helen Catalina Rabanal-León}

Docente universitaria. Magister en Psicología Educativa. Lic. en Educación especialidad Matemática.

Lic. en Administración. Universidad César Vallejo. Trujillo, Perú.

E-mail: hrabanal@ucv.edu.pe

\section{Nelly Roxana Carranza Yuncor}

Docente universitaria. Universidad César Vallejo. Trujillo, Perú.

E-mail: ncarranzay25@ucvvirtual.edu.pe

\section{Janeth Leticia Mora Zapater}

Docente universitaria. Magister en Enseñanza de Inglés como Idioma Extranjero. Licenciada en Lengua Inglesa Especialización Lingüística y Literatura. Universidad Laica Vicente Rocafuerte de Guayaquil. Guayaquil, Ecuador.

E-mail: jmoraz@ulvr.edu.ec.

\section{Norma Alexandra Hinojosa Garcés}

Docente universitaria. Máster en Gerencia y Liderazgo Educativo. Licenciada en Educación Parvularia. Universidad Laica Vicente Rocafuerte de Guayaquil. Guayaquil, Ecuador.

E-mail: normyhinojosa@gmail.com 


\title{
RESUMEN
}

Resulta necesario destacar la importancia que se da actualmente, al uso del software libre en la creación de las revistas científicas, a nivel mundial. Se planteó el objetivo general, analizar el estado del arte del software libre y las revistas científicas. Metodología, la investigación presentó un diseño cualitativointerpretativo, de tipo documental, se han seleccionado 27 documentos, realizados en el periodo 2016 2021; incluyendo: artículos científicos, artículos de revisión e información de sitios web de organizaciones reconocidas. Las palabras claves utilizadas en las búsquedas fueron: software libre, revistas científicas y OJS. Resultados, el OJS (Open Journal Systems), es un software libre que nos facilita la gestión de una revista científica. Conclusiones, la UNESCO apoya decididamente el acceso abierto al conocimiento científico, es posible descargar cualquier versión del OJS desde el Open Journal Systems Download Center, las universidades públicas y privadas pueden aprovechar el OJS, para la creación de sus revistas científicas, con una minima inversión.

Palabras clave: Software libre, revistas científicas y OJS.

\begin{abstract}
It is necessary to highlight the importance that is currently given to the use of free software in the creation of scientific journals, worldwide. The general objective was raised, to analyze the state of the art of free software and scientific journals. Methodology, the research presented a qualitative-interpretive design, documentary type, 27 documents have been selected, made in the period 2016 - 2021; including: scientific articles, review articles and information from websites of recognized organizations. The keywords used in the searches were: free software, scientific journals and OJS. Results, the OJS (Open Journal Systems), is a free software that facilitates the management of a scientific journal. Conclusions, UNESCO strongly supports open access to scientific knowledge, it is possible to download any version of the OJS from the Open Journal Systems Download Center, public and private universities can take advantage of the OJS, for the creation of their scientific journals, with a minimum investment.
\end{abstract}

Keywords: Free software, scientific journals and OJS.

\section{INTRODUCCIÓN}

Software libre es el software que respeta la libertad de los usuarios y la comunidad. A grandes rasgos, significa que los usuarios tienen la libertad de ejecutar, copiar, distribuir, estudiar, modificar y mejorar el software. Es decir, el software libre es una cuestión de libertad, no de precio (GNU, 2021).

Según Stallman (2020) las cuatro clases de libertad para los usuarios de software:

- Libertad 0: la libertad para ejecutar el programa sea cual sea nuestro propósito.

- Libertad 1: la libertad para estudiar el funcionamiento del programa y adaptarlo a tus necesidades —el acceso al código fuente es condición indispensable para esto.

- Libertad 2: la libertad para redistribuir copias y ayudar así a tu vecino.

- Libertad 3: la libertad para mejorar el programa y luego publicarlo para el bien de toda la comunidad —el acceso al código fuente es condición indispensable para esto.

Las ventajas del software libre son: Comunitario, el software libre se crea en comunidades horizontales de carácter cooperative...; seguridad, dado que el código de programación del software libre 
es abierto, el usuario puede conocer cómo está hecho el programa que usa...; gratuito, para garantizar las libertades 0 y 2 , generalmente el software libre se distribuye de forma gratuita...; interoperabilidad, el uso de formatos abiertos y estándares fomenta la interoperabilidad entre los usuarios y soberanía, el software libre otorga un mayor poder de control sobre la información y los datos con que se trabaja... (cienciassociales, 2016).

Las comunidades de colaboración en línea conllevan aprendizaje, como es el caso del software libre, y se convierten en un factor habilitador clave para lograr dichos objetivos (Gutiérrez, Bordas \& Piñón, 2020).

Se examina las experiencias de Venezuela y Ecuador como pioneros en América Latina en las políticas de apoyo a la cultura libre, uso del software libre y el progresivo empoderamiento digital del poder popular o ciudadano mediante el uso de las Tecnologías de Información y Comunicación (TIC) (Freire, Díaz \& Vera, 2019).

Los intentos de legislar el software libre en Colombia y, de manera más amplia, en América Latina, constituyen episodios sobre la construcción de la política tecnológica y sus implicaciones para el orden social (Pereira-García, 2020).

La investigación científica termina con la publicación del artículo científico; solo así pasará a formar parte del conocimiento científico (Lam, 2016).

Toda la investigación científica es crítica por definición, porque lo nuevo siempre niega dialécticamente lo anterior. Por eso podemos encontrar elementos críticos en cualquier modalidad de artículo o contribución, pero esta propuesta se limita a la crítica que se hace a un artículo previamente publicado (Castillo-González \& Dorta-Contreras, 2017).

La evolución que ha sufrido la comunicación científica en los últimos años, provocada, principalmente, por la digitalización de las publicaciones, el oligopolio de las editoriales académicas ( 5 editores controlan un 50\% del mercado), el acceso abierto y la eterna

presión por publicar a la que vive sometida el personal de investigación (Gil, 2018).

Las revistas científicas, a pesar de la jerga disciplinar en que a veces se expresan las ideas en los artículos que publican, permiten difundir, hacer llegar el conocimiento más allá de las fronteras de las instituciones, de las redes, de los países en los que el conocimiento se genera (Girola, 2019).

Es indudable que actualmente las revistas tienen una cantidad de funciones fundamentales e importantes en la producción, validación, preservación y diseminación del conocimiento (Vessuri, 2021).

Cada revista debe idealizar cual es su lector, que en todo caso pertenece a un área especializada del conocimiento, que aspira a actualizarse en temas específicos, conocer el estado de desarrollo de su área y tener un acercamiento con sus profesores y sus pares académicos (Palacios, 2016). 
Sin embargo, la relevancia de las revistas es percibida históricamente mediante dos indicadores: presencia y desempeño en índices bibliográficos de referencia internacional y por la contribución a la comunicación de las investigaciones nacionales (Packer, 2016).

La difusión del uso de software libre en el ámbito investigador de habla hispana sería una práctica altamente recomendable por parte de los principales instrumentos de transferencia de la información científica, como son las revistas científicas, especialmente aquellas que defienden la práctica de la ciencia abierta y el libre acceso al conocimiento científico, como Gaceta Sanitaria (Prieto-Botella, Valera-Gran \& Navarrete-Muñoz, 2020).

Uno de estos grandes aportes a la ciencia lo constituye las herramientas implementadas en el mundo para la edición de las revistas científicas, entre ellas la plataforma OJS (Open Journal Systems), muy utilizada en la actualidad y más necesaria cada día en el desarrollo de los procesos editoriales, al agilizarlos y, así, reducir el tiempo de publicación (Acosta, Cascaret, Pardo, Izquierdo y Mustelier (2019).

De modo que, en 2001, nace OJS como iniciativa de la Universidad de Columbia Británica, el Consejo de Investigación en Ciencias Sociales y Humanidades de Canadá, la Fundación Max Bell, la Pacific Press Endowment y la Fundación MacArthur con el proyecto Public Knowledge Project (PKP) (Vizcaino, 2020).

OJS está diseñado para ser un sistema multirevista. El administrador/a del sitio web es responsable de configurar las características de sus distintos niveles y de crear nuevas revistas que se almacenen dentro de un mismo sitio web. Los sitios web de las revistas son totalmente independientes, a excepción de las cuentas de los usuarios/as (PoliPapers - UPV (2021).

OJS incluye las siguientes características: OJS se instala localmente y se controla localmente, los editores configuran requisitos, secciones, proceso de revisión, etc., envío en línea, revisión doble ciego y administración de todo el contenido, amplia indexación del contenido, interfaz de lectura con capacidad de respuesta y temática, notificación por correo electrónico para los lectores, soporte para cargos de procesamiento de artículos, suscripciones y pagos en línea, completo soporte de ayuda en línea sensible al contexto, soporte multilingüe (PKP, 2021). 
Figura 1. Open Journal Systems Download Center

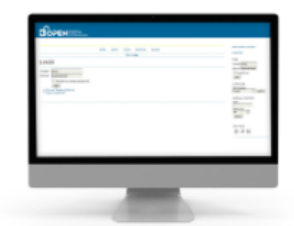

Latest Version: 3.3.0-3 | Release Date: Feb 2021

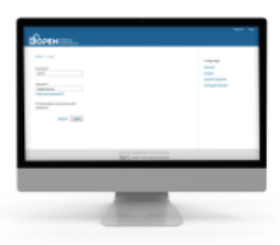

Latest Version: 3.2.1-4 | Release Date: Feb 2021
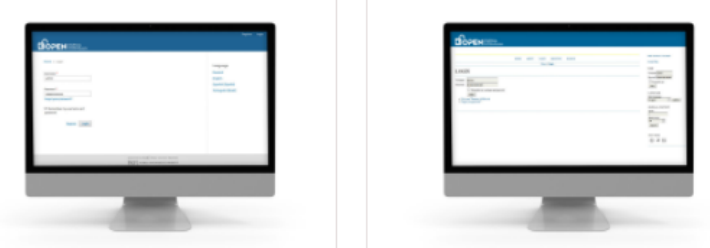

Latest Version: 3.1.2-4 | Release Date: Dec 2019 2019

Figura 2. Proceso Editorial y Publicación OJS.

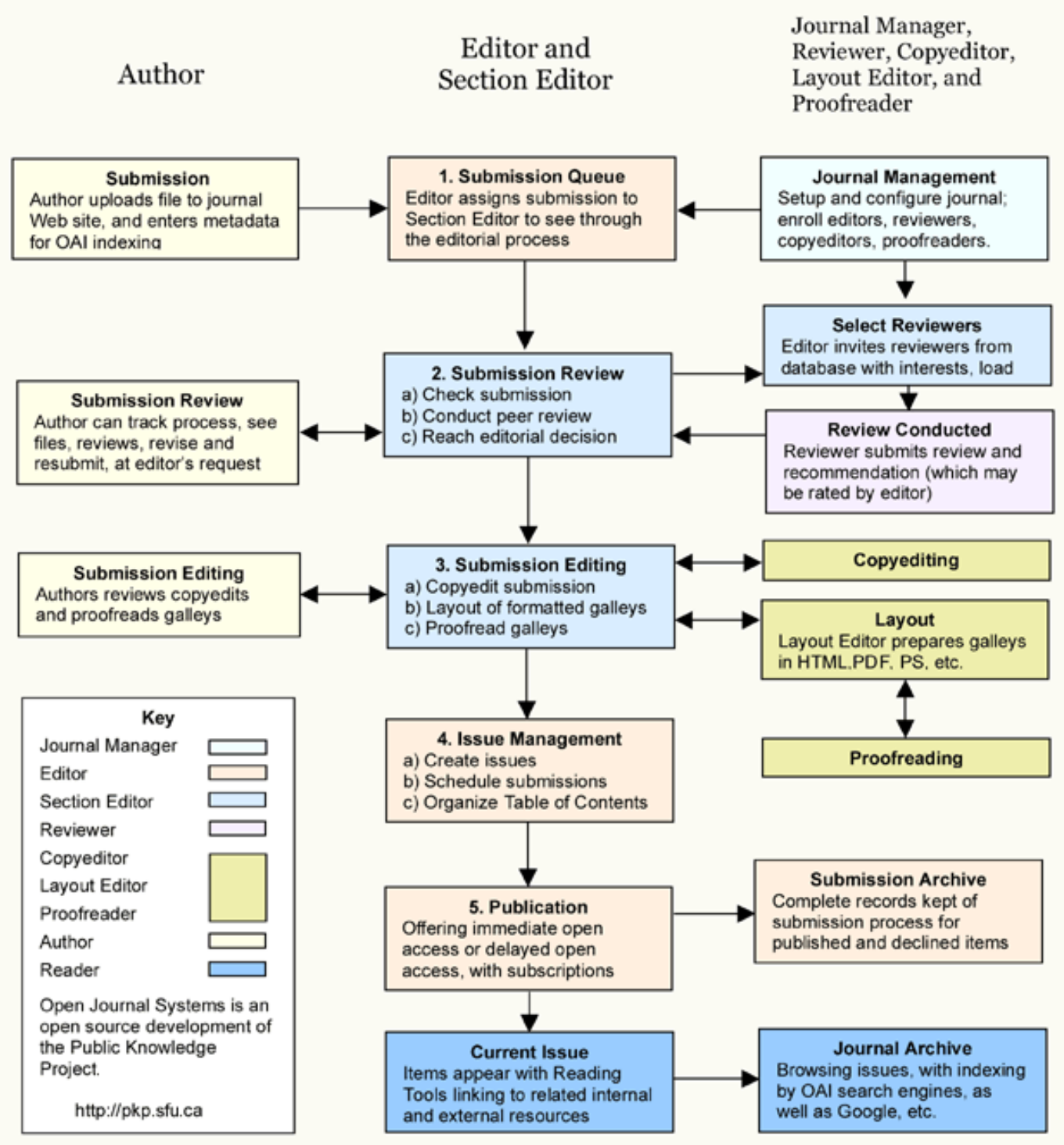

Fuente: YACHAQ (2021) 
El Sistema de Revistas Abiertas (OJS, por sus siglas en inglés) es un sistema de administración y publicación de revistas académicas desarrolladas por el Proyecto de Conocimiento Público, Canadá (PKP, por sus siglas en inglés). El sistema está diseñado para reducir el tiempo y esfuerzo necesario para la edición de una revista académica (Alperin, 2017).

PKP y SciELO planean colaborar en la construcción de un sistema de Servidor de Preprints totalmente interoperable con Open Journal System (OJS) y otros sistemas de publicación que servirán a las revistas de la Red SciELO y que se estarán disponibles al público para que operen con otras organizaciones (SciELO, 2018).

En referencia a la ciencia abierta, se expusieron aspectos de accesibilidad y de disponibilidad con el objetivo de sostener la naturaleza académica y abierta de la comunicación científica sin barreras de ningún tipo (Garrido, Ruiz \& Bosi, 2021).

La UNESCO ha apoyado los esfuerzos para el acceso abierto al conocimiento científico a través de nuestros socios como la Federación Internacional de Asociaciones e Instituciones de Bibliotecarios (IFLA). La UNESCO apoya el llamamiento de IFLA y sus asociados para garantizar el libre acceso a la información científica, en particular en este momento crucial en que el mundo debe trabajar colectivamente para encontrar vacunas y tratamientos contra el virus Covid-19 (UNESCO, 2020).

\section{METODOLOGÍA}

La investigación presentó un diseño cualitativo-interpretativo, de tipo documental, el cual precisó el procedimiento de selección y el registro de los datos (Barrero y Rosero, 2018).

En la presente investigación se seleccionaron 27 documentos, realizados en el periodo 2016 2021; incluyendo: artículos científicos, artículos de revisión e información de sitios web de organizaciones reconocidas. Las palabras claves utilizadas en las búsquedas fueron: software libre, revistas científicas y OJS. Para la selección de los documentos se usaron como criterios: el año de la publicación, la pertenencia con la investigación y ser una fuente confiable.

Después de la lectura de cada documento, se procedió a ingresar los datos a la matriz bibliográfica, que sirvió para catalogar los documentos de acuerdo con categorías, que se presentan en la tabla 1.

Tabla 1. Matriz bibliográfica

\begin{tabular}{|l|l|l|l|}
\hline Nombre & Tipo & Objetivos & Conclusiones \\
\hline & & & \\
\hline & & & \\
\hline
\end{tabular}

Fuente: Adaptado de Barrero y Rosero (2018). 


\section{RESULTADOS}

Actualmente, OJS está siendo utilizada por más de 10.000 revistas en el mundo, con un gran porcentaje de ellas en América Latina, donde se encuentran al menos 3.250 revistas que publicaron 10 documentos o más en 2015, incluyendo a 161 revistas de la Universidad de São Paulo y a 85 revistas de la Universidad Autónoma de México. Colectivamente, estás revistas han publicado más de 400.000 documentos (Alperin, 2017).

Se encontró 351 revistas en la revisión de cada portal, se excluyó 27 títulos que no tenían ningún artículo publicado en el portal de OJS y se analizó solo 324 journals...Todas las revistas editadas por las universidades peruanas encontradas, son

de acceso abierto. El porcentaje de las que son publicadas por instituciones nacionales es menor (29\%) que las editadas por instituciones particulares (71\%) (Yance-Yupari, 2018).

Las revistas científicas de Ecuador, que utilizan OJS, se muestran en la tabla 2. Se encontró cuatro revistas científicas que utilizan las versiones 3.1.2.1, 3.2.0.3 y 3.2.1.1 del OJS.

Tabla 2. Revistas científicas de Ecuador que utilizan OJS.

\begin{tabular}{|c|c|c|c|c|}
\hline Institución & Nombre de la revista & URL & ISSN & $\begin{array}{l}\text { Versión del } \\
\text { OJS }\end{array}$ \\
\hline $\begin{array}{l}\text { Universidad Laica } \\
\text { Vicente Rocafuerte de } \\
\text { Guayaquil }\end{array}$ & $\begin{array}{l}\text { Yachana Revista } \\
\text { Científica }\end{array}$ & $\begin{array}{l}\text { http://revistas.ulvr.edu.ec/inde } \\
\text { x.php/yachana }\end{array}$ & $1390-7778$ & 3.2 .0 .3 \\
\hline $\begin{array}{l}\text { Universidad } \\
\text { Tecnológica } \\
\text { Empresarial } \\
\text { Guayaquil } \\
\end{array}$ & $\begin{array}{l}\text { Revista Ciencia \& } \\
\text { Tecnología }\end{array}$ & $\begin{array}{l}\text { http://cienciaytecnologia.uteg. } \\
\text { edu.ec/revista/index.php/cienc } \\
\text { iaytecnologia }\end{array}$ & $2661-6734$ & 3.2 .1 .1 \\
\hline $\begin{array}{l}\text { Universidad Politécnica } \\
\text { Salesiana de Ecuador }\end{array}$ & Alteridad & $\begin{array}{l}\text { https://revistas.ups.edu.ec/inde } \\
\text { x.php/alteridad }\end{array}$ & $1390-8642$ & 3.1 .2 .1 \\
\hline $\begin{array}{l}\text { Universidad Técnica de } \\
\text { Machala }\end{array}$ & Cumbres & $\begin{array}{l}\text { https://investigacion.utmachal } \\
\text { a.edu.ec/revistas/index.php/Cu } \\
\text { mbres }\end{array}$ & $1390-9541$ & 3.2 .1 .1 \\
\hline
\end{tabular}

Fuente: Elaboración propia.

El recurso más importante para conocer las revistas de acceso abierto es el Directory of Open Access Journals (DOAJ). Este índice recoge más de 14.000 revistas OA de calidad, revisadas por pares, que cubren todas las áreas de Ciencia, Medicina, Tecnología, CC. Sociales y Humanidades. El Directory of Open Access Journals (DOAJ) lista revistas de acceso libre, científicas y académicas, que cumplan con estándares de alta calidad al utilizar la revisión por pares o control de calidad editorial y que sean gratuitas para todos al momento de su publicación, sobre la base de la definición de acceso libre adoptada en la Budapest Open Access Initiative (Biblioteca de la Universidad de Huelva, 2021). 
Esto demuestra la posibilidad de creación y mantenimiento de una revista científica de acceso abierto desde una Institución de Educación Superior angolana, usando el Open Journal System como herramienta para la difusión del conocimiento, así como el avance que se puede tener en el corto y mediano plazo en la internacionalización de la misma, tomando las decisiones adecuadas (Artigas \& Wongo, 2020).

El OJS (Open Journal Systems), es un software libre que nos facilita la gestión de una revista científica, como se muestra a continuación.

Figura 3. Ingreso al OJS para configurar la revista científica.

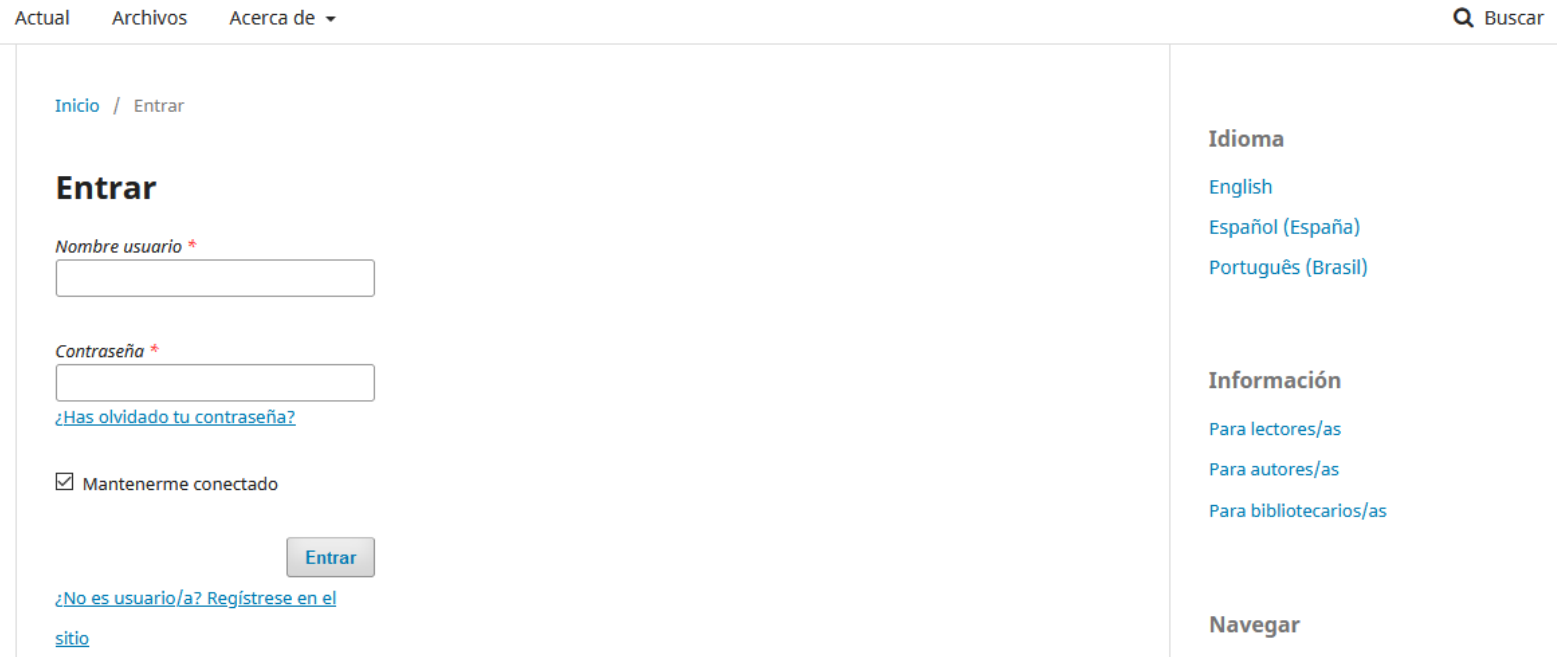

Fuente: Elaboración propia.

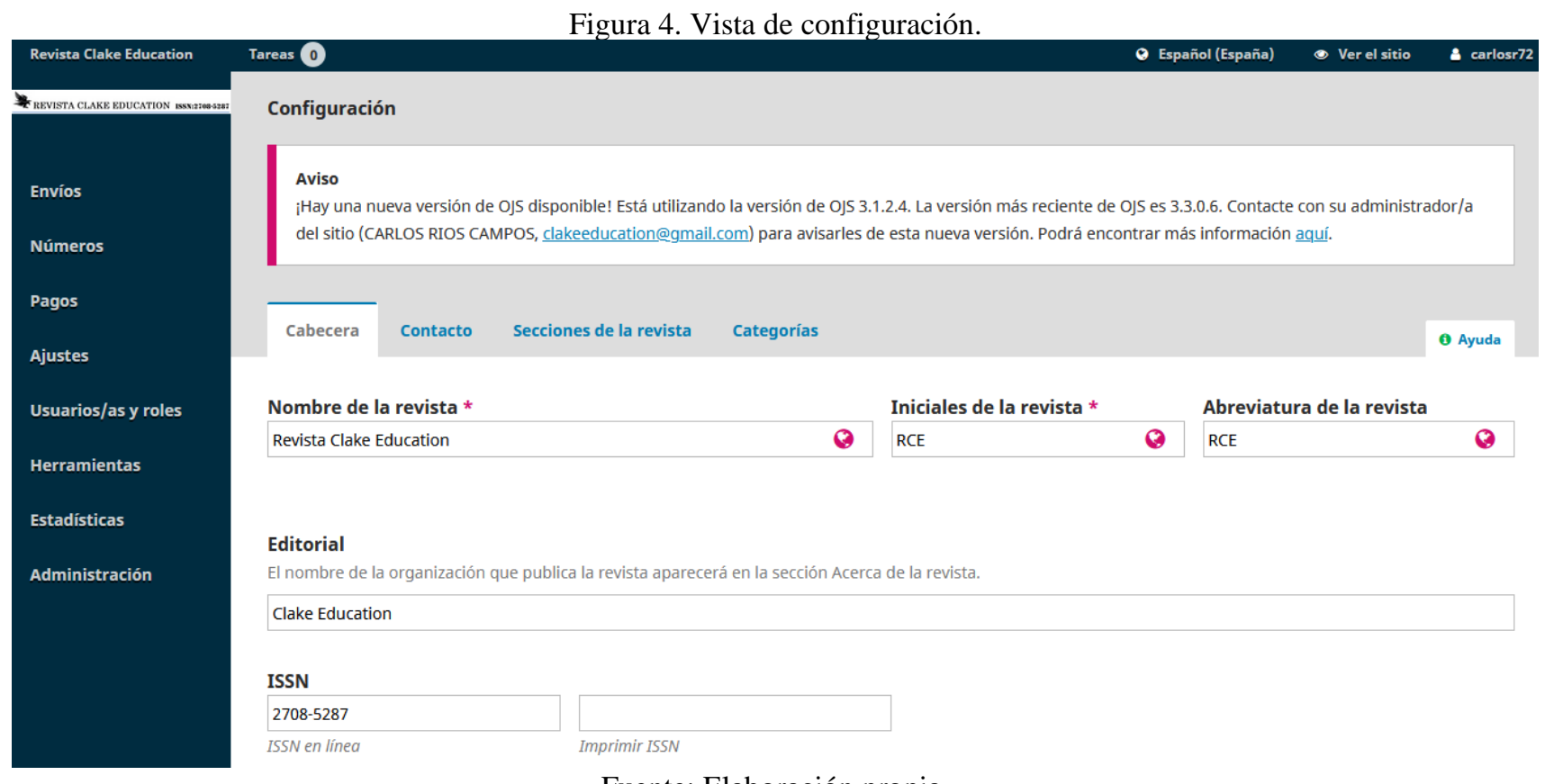

Fuente: Elaboración propia. 
Figura 5. Visualizar los números de la revista científica.

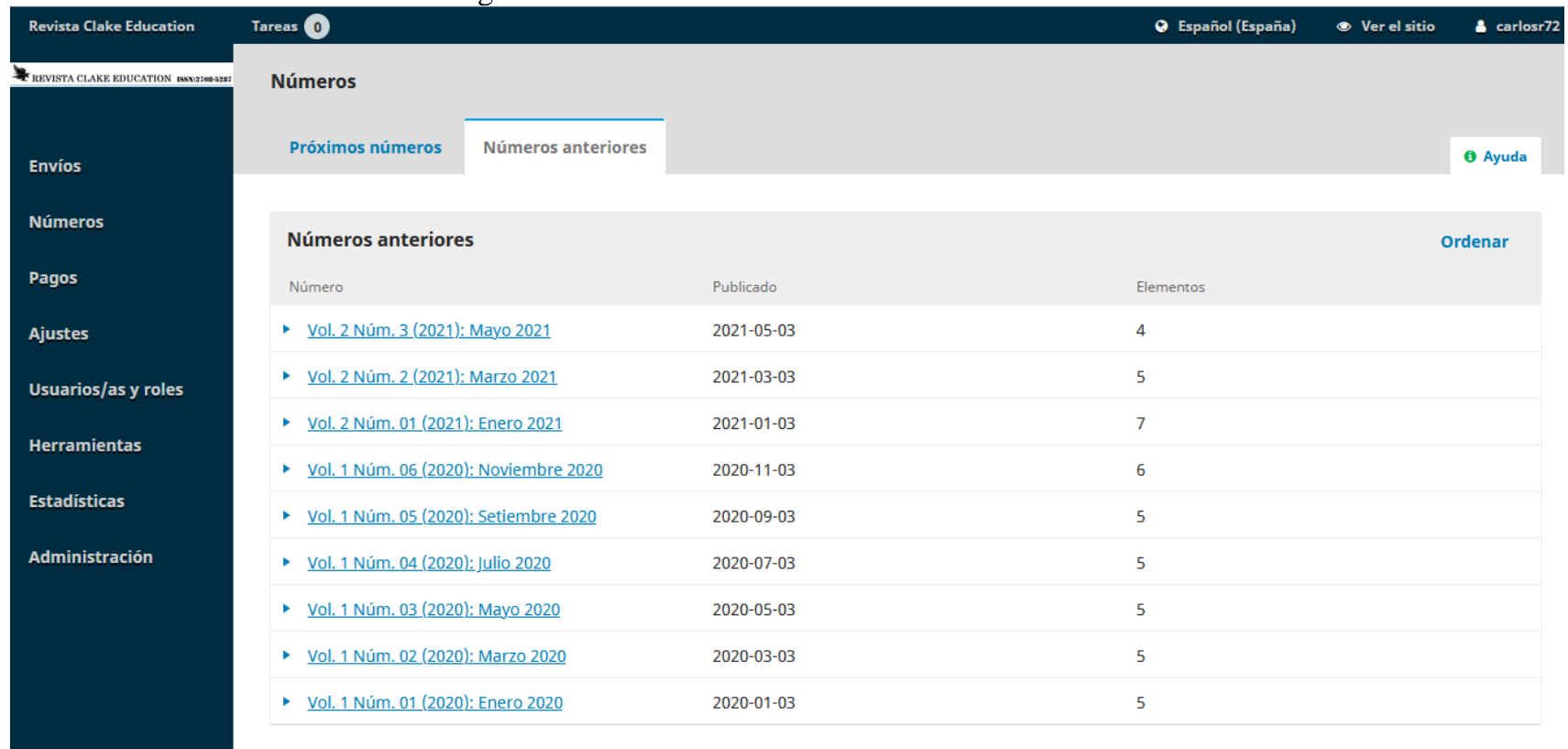

Fuente: Elaboración propia.

Figura 6. Configurar los pagos y suscripciones.

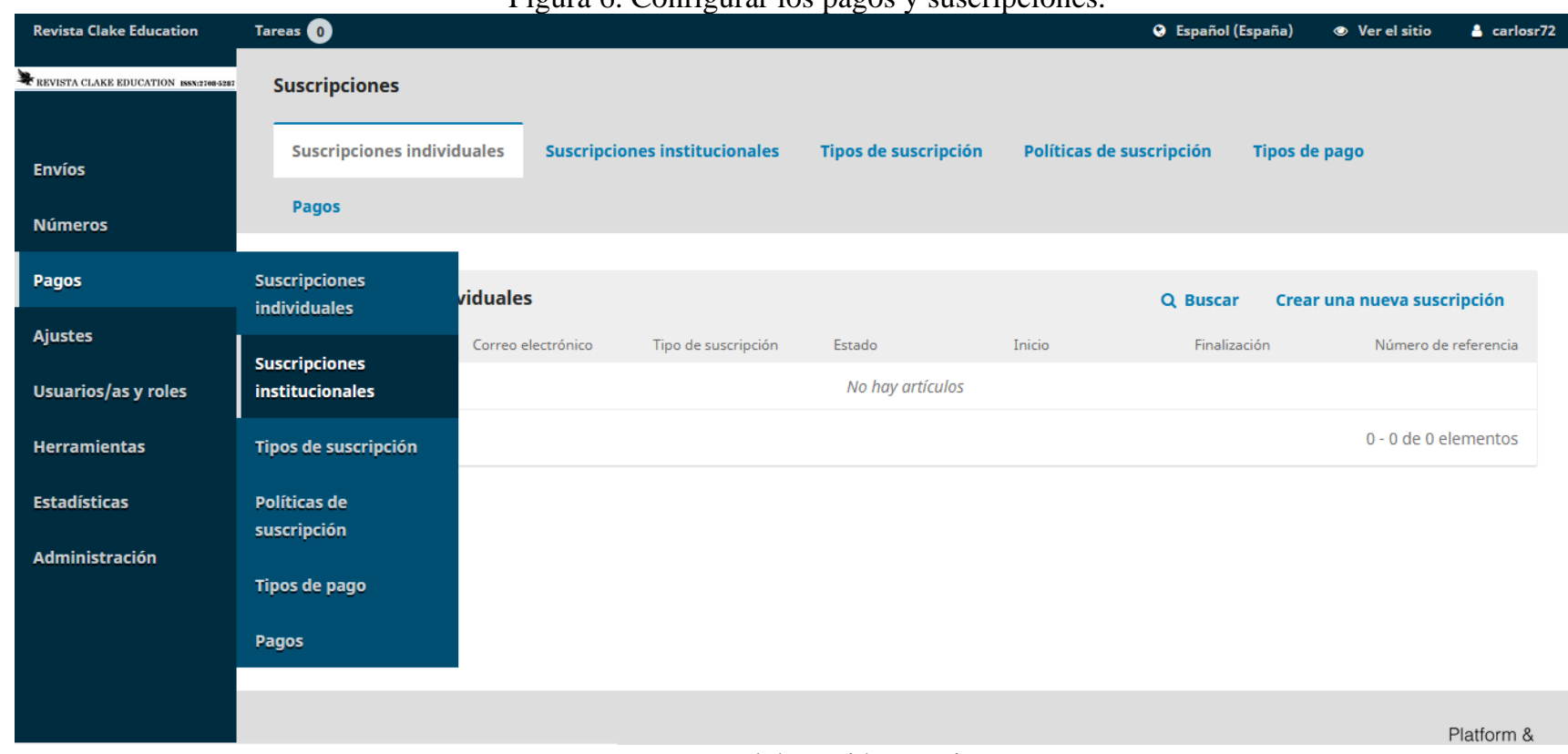

Fuente: Elaboración propia. 
Figura 7. Ajustes de la revista, sitio web, flujo de trabajo y distribución.

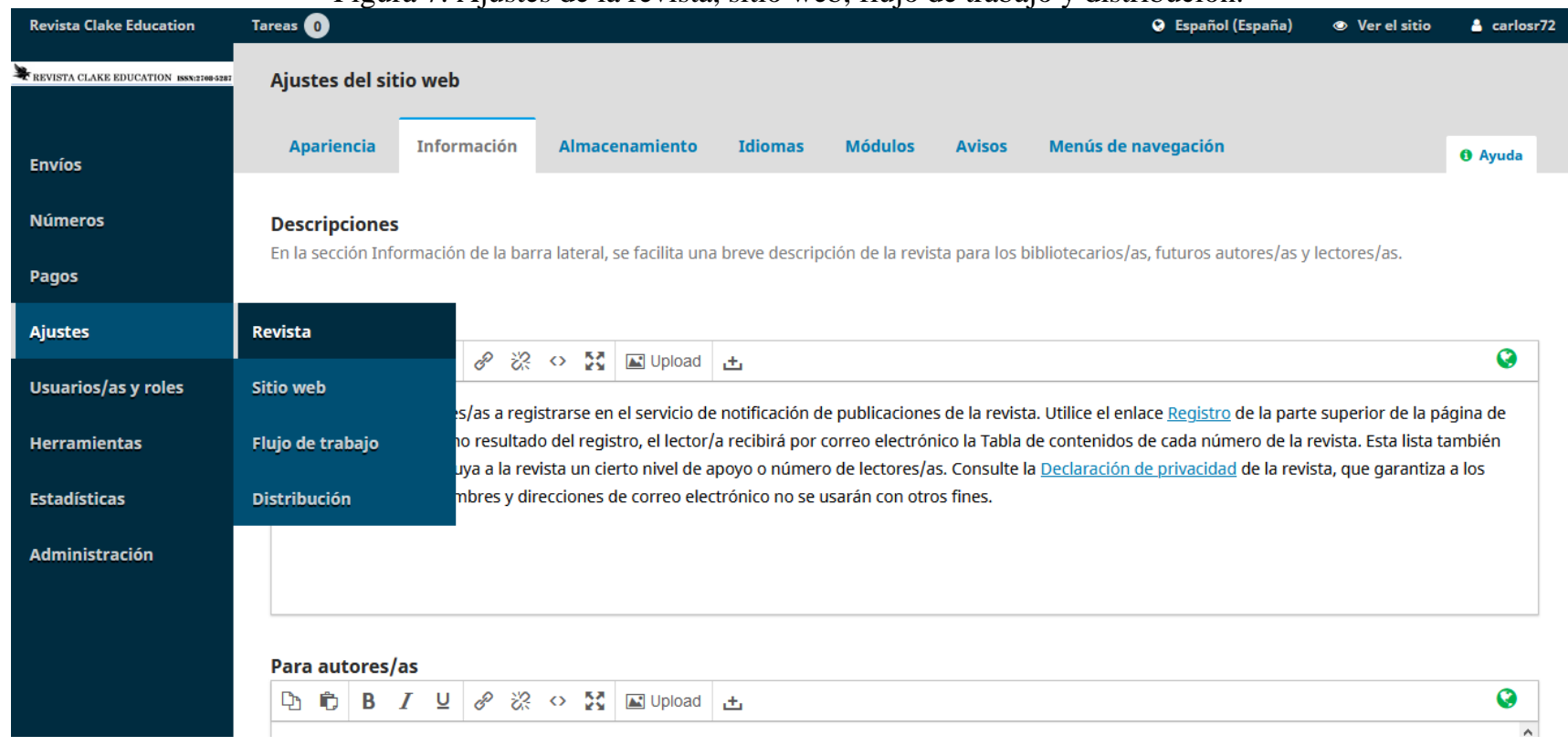

Fuente: Elaboración propia.

Figura 8. Configuración de usuarios, tareas y opciones de acceso a sitios.

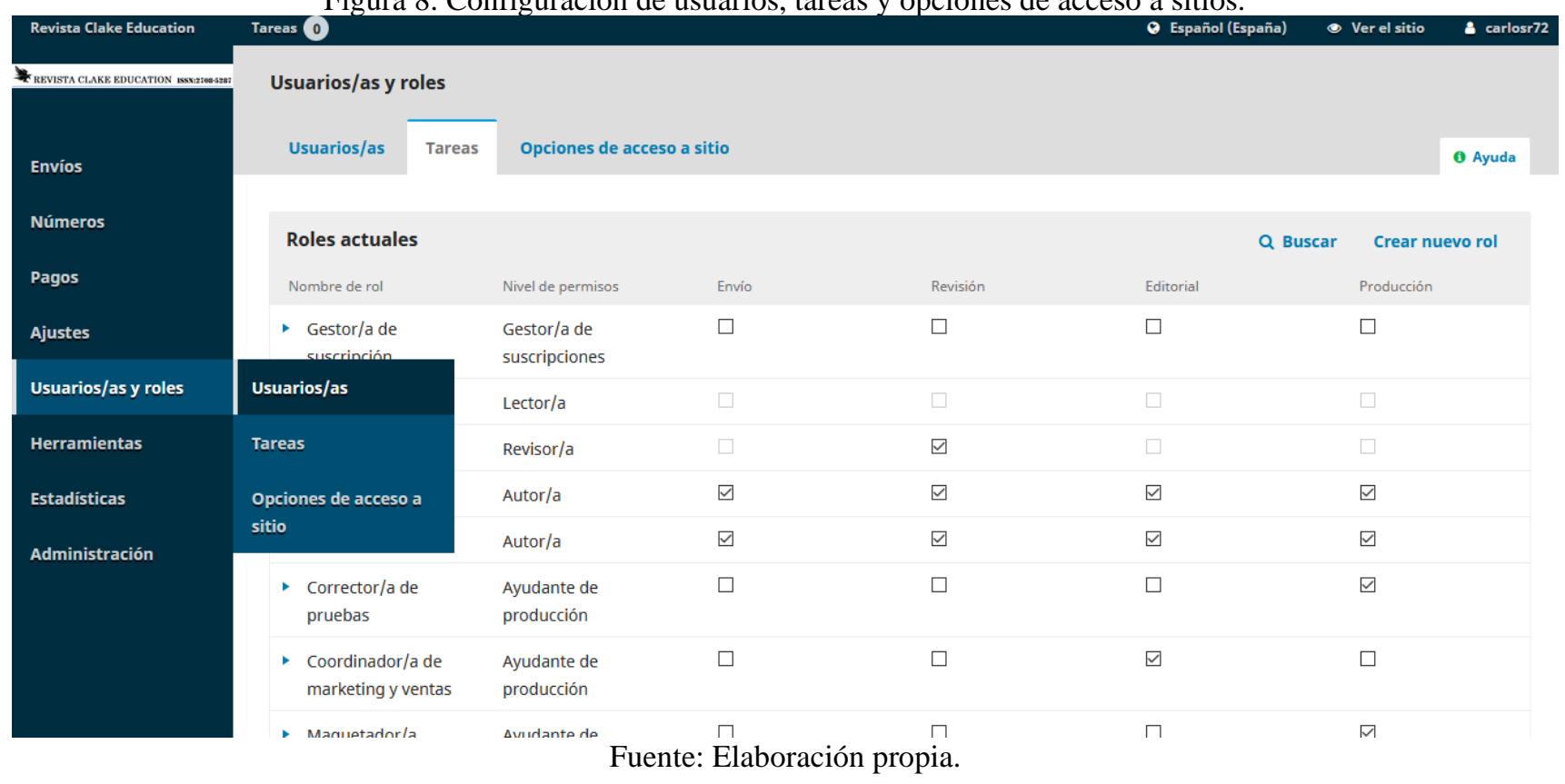


Figura 9. Herramientas de importar/exportar y generador de informes.

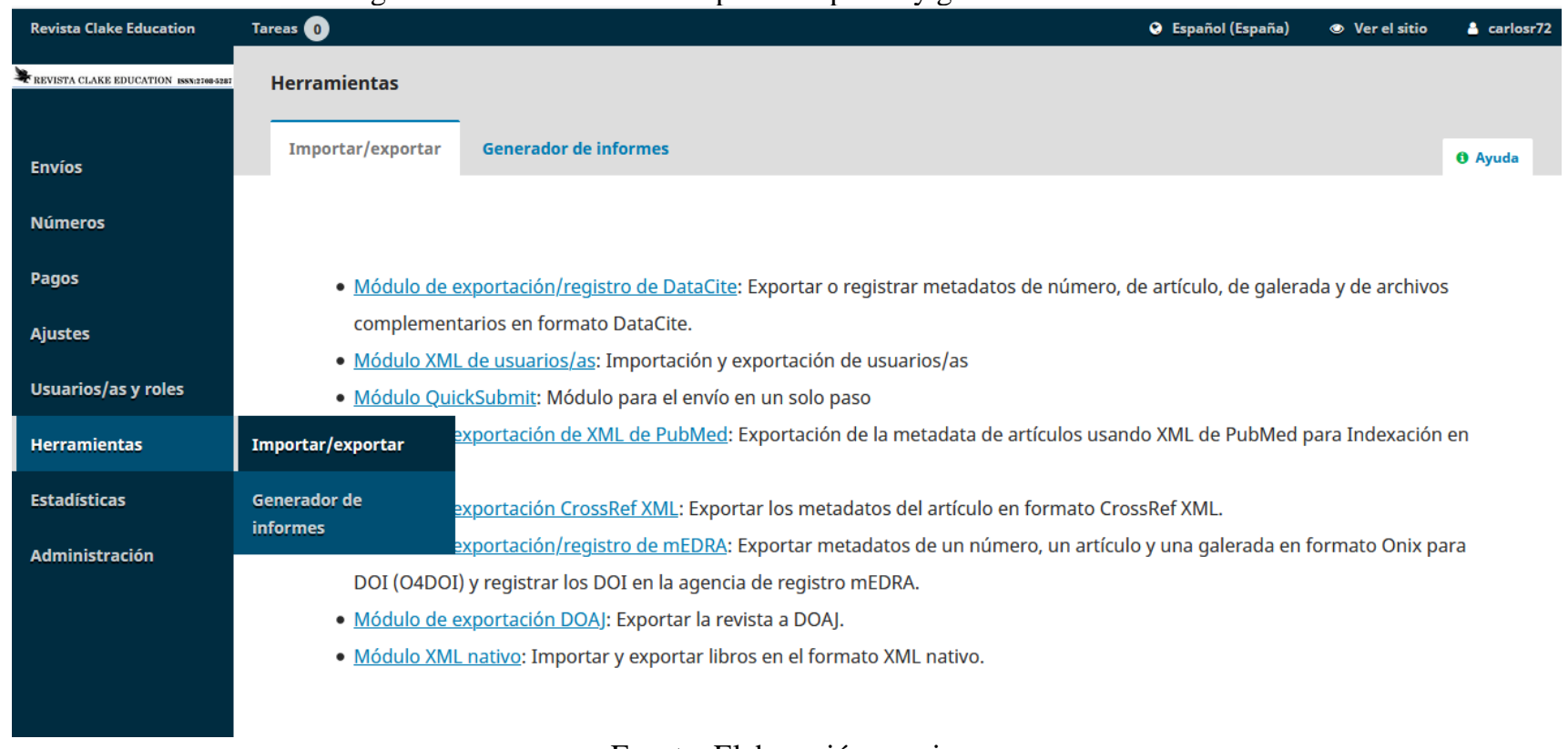

Fuente: Elaboración propia.

Figura 10. Módulo QuickSubmit.

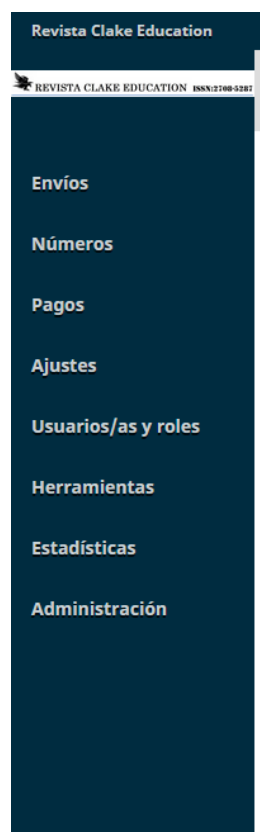

\section{Tareas 0}

Módulo QuickSubmit

Este módulo permite enviar rápidamente envíos a la etapa de producción o directamente a un número.

Imagen de portada

\pm Subir

Idioma del envío

Español (España)

Se aceptan envíos en varios idiomas. Elija el idioma principal del envío desde el menú

desplegable de abajo. *

Sección

seleccione la sección adecuada para el envio (ver Secciones y Politica en Acerce de lo

revista). *

Prefijo

Título *

Si el título del libro comienza con "Un/a"

o "FI/I a/I Q Q /I as" (o alan similar que no

Fuente: Elaboración propia. 
Figura 11. Estadísticas.

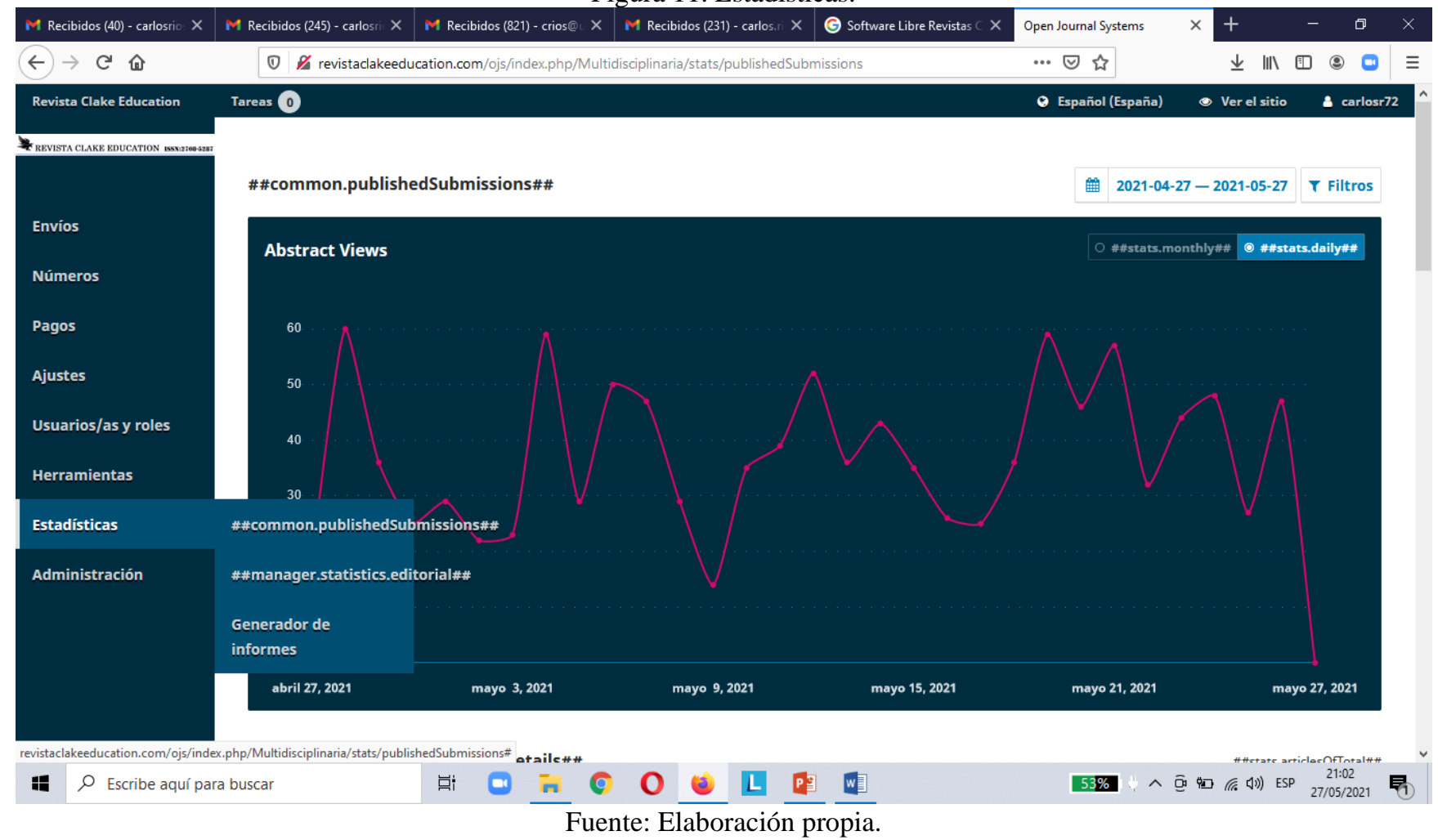

Figura 12. Administración (Gestión del sitio y funciones administrativas).

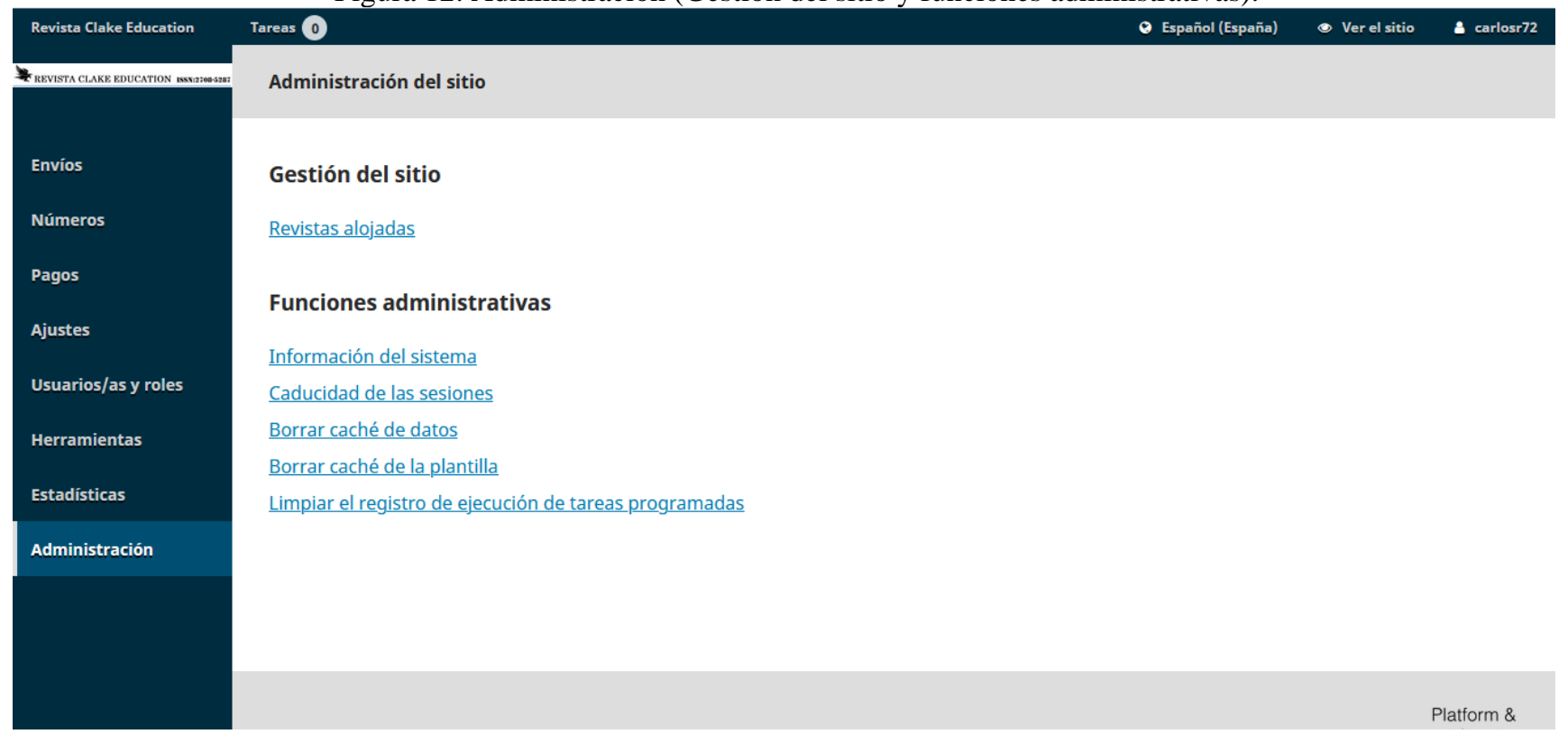

Fuente: Elaboración propia.

El OJS (Open Journal Systems), es un software que dispone de muchas herramientas que simplifican el trabajo del editor de la revista científica.

\section{CONCLUSIONES}

La UNESCO apoya decididamente el acceso abierto al conocimiento científico. 
El OJS es un software libre, muy útil en la gestión de las revistas científicas. Es utilizado a nivel mundial por la facilidad de uso, por su acceso libre al código y por la comunidad de soporte al mismo.

Es posible descargar cualquier versión del OJS desde el Open Journal Systems Download Center.

Las universidades públicas y privadas pueden aprovechar el OJS, para la creación de sus revistas científicas, con una minima inversión. 


\section{REFERENCIAS BIBLIOGRÁFICAS}

Acosta, L., Cascaret, X., Pardo, M., Izquierdo, J. y Mustelier, H. (2019). Open Journal Systems: resultados de la implementación de videos tutoriales como iniciativa para el proceso editorial de una revista. MEDISAN, vol. 23, núm. 6, pp. 1104-1113, 2019

Alperin, J. (2017). ¿Cómo el software libre apoya la diseminación del conocimiento?. Recuperado de https://blogs.iadb.org/conocimiento-abierto/es/como-el-software-libre-apoya-la-diseminacion-delconocimiento/

Artigas, Wileidys, \& Gungula, Eurico Wongo. (2020). Gestión de revistas a través de OJS: Experiencia de éxito en Angolana. E-Ciencias de la Información, 10(1), 1-18. https://dx.doi.org/10.15517/eci.v10i1.39771

Barrero, A. y Rosero, A. (2018). Estado del Arte sobre Concepciones de la Diversidad en el Contexto Escolar Infantil. Revista Latinoamericana de Educación Inclusiva, 2018, 12(1), 39-55 https://doi.org/10.4067/S0718-73782018000100004

Biblioteca de la Universidad de Huelva (2021). Acceso abierto a la producción científica: Publicar en revistas OA. Recuperado de https://guiasbuh.uhu.es/c.php?g=432343\&p=3384143

Castillo-González, W. \& Dorta-Contreras, A. (2017). Crítica científica. Una propuesta metodológica. Educación Médica, Volume 18, Issue 4, Pages 285-288, ISSN 1575-1813. https://doi.org/10.1016/j.edumed.2016.10.001.

Ciencias sociales (2016). Qué es Software. Recuperado de https://cienciassociales.edu.uy/que-es-elsoftware-libre/

Freire Avilés, R. M., Díaz Nava, J. B., \& Vera Lucio, N. E. (2019). Cultura libre y software libre: hacia el empoderamiento digital. Revista Prisma Social, (26), 50-72. Recuperado a partir de https://revistaprismasocial.es/article/view/3115

Garrido, A., Ruiz, A., \& Bosi García, S. F. (2021). Del software libre al acceso abierto: repercusiones en la visibilidad de la producción científica. Investigación Joven, 7(2), 706-707. Recuperado a partir de https://revistas.unlp.edu.ar/InvJov/article/view/11388

Gil, L. (2018). Reseña Gestión y edición de revistas académicas con software libre. El uso de Open Journal Systems 3. Ángel M. Delgado-Vázquez. 2018. Anales de Documentación, 21(2). Recuperado a partir de https://revistas.um.es/analesdoc/article/view/345021

Girola Molina, Lidia Graciela. (2019). Las revistas científicas como instrumento de difusión del conocimiento. Revista mexicana de sociología, 81(4), 919-927. Epub 07 de noviembre de 2019.https://doi.org/10.22201/iis.01882503p.2019.4.57982

GNU (2021). ¿Qué es el software libre?. Recuperado de https://www.gnu.org/philosophy/free-sw.es.html

Gutiérrez Diez, María del Carmen, Bordas Beltrán, José Luis, \& Piñón Howlet, Laura Cristina. (2020). Factores motivacionales en los desarrolladores de software libre. Investigación administrativa, 49(126), 12604. Epub 01 de septiembre de 2020.https://doi.org/10.35426/iav49n126.04 
Lam Díaz, Rosa María (2016). La redacción de un artículo científico. Revista Cubana de Hematología, Inmunología y Hemoterapia, 32(1), 57-69. Recuperado de http://scielo.sld.cu/scielo.php?script=sci_arttext\&pid=S0864-02892016000100006\&lng=es\&tlng=es.

Openjournalsystems.com (2021). Open Journal Systems Download Center. Recuperado de https://openjournalsystems.com/ojs-download/

Packer, A. (2016). SciELO y el futuro de las revistas. SciELO en Perspectiva. Available from: https://blog.scielo.org/es/2016/12/21/scielo-y-el-futuro-de-las-revistas/

Palacios, Mauricio. (2016). ¿Cuál es el objetivo de una revista científica. Ingeniería y competitividad, 18(2), 8-10. Retrieved May 21, 2021, from http://www.scielo.org.co/scielo.php?script=sci_arttext\&pid=S0123-

$30332016000200001 \& \operatorname{lng}=\mathrm{en} \& \operatorname{tlng}=\mathrm{es}$.

Pereira-García, Alexander. (2020). Los proyectos de ley sobre software libre en Colombia. Revista Brasileira de Ciências Sociais, 35(104), e3510406. Epub May 08, 2020.https://doi.org/10.1590/3510406/2020

PKP (2021). Capítulo 1: Introducción. Recuperado de https://docs.pkp.sfu.ca/learningojs/3.1/es/introduction

PoliPapers - UPV (2021). Ayuda de Open Journal Systems. Recuperado de https://polipapers.upv.es/index.php/REDU/help/view/site/topic/000002

Prieto-Botella, Daniel, Valera-Gran, Desirée, \& Navarrete-Muñoz, Eva-María. (2020). Contribución a la práctica de ciencia abierta: el uso de software libre en Gaceta Sanitaria. Gaceta Sanitaria, 34(2), 213-214. Epub 01 de junio de 2020.https://dx.doi.org/10.1016/j.gaceta.2019.11.004

SciELO (2018). PKP y SciELO anuncian el desarrollo de un sistema de código fuente abierto de Servidor de Preprints. Recuperado de https://blog.scielo.org/es/2018/09/21/pkp-y-scielo-anuncian-el-desarrollode-un-sistema-de-codigo-fuente-abierto-de-servidor-de-preprints/\#.YKcU86G23IU

Stallman, R. (2020). La definición de software libre. Communiars. Revista de Imagen, Artes y Educación Crítica y Social, 3, 151-154.

Vessuri, H. (2018). El paper y las revistas científicas ¿tienen futuro? [online]. SciELO en Perspectiva. Available from: https://blog.scielo.org/es/2018/08/20/el-paper-y-las-revistas-cientificas-tienen-futuro/

Vizcaino, A. (2020). La gestión de OJS 3.0: Software libre preferente para Editores. https://doi.org/10.3916/club-de-editores-029

YACHAQ (2021). Acerca de este sistema de publicación. Recuperado de https://revista.uct.edu.pe/index.php/YACHAQ/about/aboutThisPublishingSystem

Yance-Yupari, V. (2018). Uso de Open Journal System en revistas científicas peruanas. Cultura, 2018, 32, 353-366 (enero - diciembre). https://doi.org/10.24265/cultura.2018.v32.16 